\title{
Resistencia química del hormigón XXVIII.-Contribución al estudio del sistema Cemento P-550-ARI hidratado-disolución de sulfato de sodio
}

M. ${ }^{\circ}$ FRANCISCA BERMEJO-MUÑOZ, JOSE L. SAGRERA-MORENO Y DEMETRIO GASPAR-TEBA IETCC/CSIC

Fecha de recepción $12 ; / / 87$

Serrano Galvache, s.n. - 28033 Madrid/España

\section{RESUMEN}

En el presente trabajo, continuación de otros, se estudia el comportamiento de un cemento portland de alta resistencia inicial ( $P$-550-ARI) con unos contenidos calculados (Bogue) de $\mathrm{C}_{3} \mathrm{~S}, \mathrm{C}_{2} \mathrm{~S}, \mathrm{C}_{3} \mathrm{~A}, \mathrm{C}_{4} \mathrm{AF}$ y $\mathrm{CaSO}_{4}$ del 39,2 - 25,7 $9.9-12,0$ y $6.9 \%$, respectivamente, cuando se somete a la acción de una disolución de sulfato de sodio $[2,1 \mathrm{~g} / \mathrm{l}$ de $\mathrm{Na}_{2} \mathrm{SO}_{4}<>1,42 \mathrm{~g} / \mathrm{l}$ de $\mathrm{SO}_{4}(\mathrm{II})<>1,48 \times 10^{-2} \mathrm{mo}-$ les/litro de $\mathrm{SO}_{4}(\mathrm{II})$ ]. que atraviesa sendos lechos granulados fabricados con dicho cemento hidratado y curado durante 7 y 28 dias, determinando:

a) la evolución del contenido de iones $\mathrm{Ca}$ (II) y $\mathrm{SO}_{4}$ (II) en las diversas fracciones recogidas de la disolución de sulfato de sodio que han atravesado los lechos de cemento, asi como la del $\mathrm{pH}$ y de la conductividad,

b) la variación de las cantidades de dichos iones, que se encuentran formando los compuestos correspondientes, en el cemento hidratado de los lechos antes y despúes de someterlos a la acción de la disolución de sulfato de sodio,

c) las modificaciones estructurales experimentadas por los compuestos cristalinos del cemento hidratado de los lechos sometidos a la acción de la disolución mencionada.

\section{SUMMARY}

In this work, which is the following of the others, the pertormance of a portland cement of a high initial resistance ( $P$-550-ARI) - with a calculated content (Bogue) of $39,2-25,7-9,9-12,0$ and $6,9 \%$ of $C_{3} S-C_{2} S-C_{3} A-C_{4} A F$ and $\mathrm{CaSO}_{4}$, respectively - is studied when it is submitted to the action of an sodium sulphate solution $[2,1 \mathrm{~g} / \mathrm{l}$ of $\mathrm{Na}_{2} \mathrm{SO}_{4}<>1,42 \mathrm{~g} / \mathrm{l}$ of $\mathrm{SO}_{4}(\mathrm{II})<>1.48 \times 10^{-2}$ moles/litre of $\mathrm{SO}_{4}(\mathrm{II})$ ] running across the beds of granulated cement. made with this hydrated cement cured during 7 or 28 days, determining:

a) The evolution of the content on ions $\mathrm{Ca}$ (II) and $\mathrm{SO}_{4}$ (II) on the taken diverse fractions of sodium sulphate solution which have run across those beds, so as this of the $\mathrm{pH}$ and of the conductivity,

b) the variation in the amount of these ions that are forming the correspondents compounds, in the hydrated cement from the beds that have been submitted before and after to the action of the sodium sulphate solution, and

c) the structural modifications undergone by the crytalline compounds of the hydrated cement from the beds submitted to the action of the sodium sulphate solution.

\section{INTRODUCCION}

En un trabajo anterior (1) se ha estudiado el comportamiento de un cemento portland industrial de alta resistencia inicial (cemento $1<>$ $<>$ P-550-ARI), análogo al del presente artículo, cuando se somete a la acción del agua desionizada (sistema: cemento P-550-ARI, hidratado-agua desionizada), que atraviesa sendos lechos granulados fabricados con dicho cemento, una vez hidratado y curado durante 7 y 28 días, determinando la evolución del pH y de la conductividad, la variación de la concentración de los iones $\mathrm{Ca}$ (II) y $\mathrm{SO}_{4}$ (II) en el agua desionizada y en el cemento del lecho, así como las modificaciones estructurales experimentadas por los compuestos cristalinos del cemento hidratado de los mencionados lechos.
Este cemento $1<>$ P-550-ARI es, teóricamente, análogo al utilizado en otros trabajos, de cuyos resultados se viene dando cuenta en diversos artículos (2) (3) (4) (5) (6) (7), en donde se estudia el comportamiento de las probetas de mortero $(1: 3)$, fabricadas con dicho cemento 1 , cuando - una vez curadas en agua potable filtrada- se someten a la acción del agua potable tiltrada, del agua de mar artificial ASTM D 1141-75 y de una disolución saturada de yeso, en donde se han sumergido durante diversos períodos de tiempo, o cuando se hace pasar agua de mar artificial ASTM a través de un lecho de mortero (8).

El presente trabajo, que forma parte de un amplio Programa de Investigación del I.E.T.c.c., se ha realizado para contribuir al conocimiento de la resistencia química de los cementos, en 
general, frente a la acción de diversas disoluciones agresivas, de cuyos resultados se viene dando cuenta en diversos artículos.

\section{PARTE EXPERIMENTAL}

En el presente trabajo se estudia el comportamiento de un cemento portland industrial de alta resistencia inicial (P-550-ARI), designado cemento 1, cuando los lechos fabricados con dicho cemento, una vez que se ha hidratado, granulado y conservado durante 7 ó 28 días en un recinto con una humedad relativa superior al $90 \%$, a una temperatura de $21 \pm 2^{\circ} \mathrm{C}$, se someten a la acción de una disolución acuosa de sulfato de sodio (sistema: cemento 1 hidratado-disolución de sulfato de sodio), determinando:

a) la concentración de los iones $\mathrm{Ca}$ (II) y $\mathrm{SO}_{4}$ (II) tanto en cada una de las fracciones recogidas de la disolución, una vez que han atravesado los lechos, como en el cemento hidratado del lecho, antes y despúes de haber pasado por el mismo la disolución de sulfato de sodio,

b) la evolución del $\mathrm{pH}$ y de la conductividad de las fracciones recogidas de la disolución de sulfato de sodio, que han atravesado los lechos de cemento,

c) las modificaciones estructurales experimentadas por los compuestos cristalinos del cemento hidratado del lecho, sometido a la acción de la disolución de sulfato de sodio, con relación a la composición estructural del cemento hidratado.

\subsection{Materiales utilizados}

\section{a) Cemento 1}

Se ha utilizado el cemento portland industrial de alta resistencia inicial (cemento $1<>$ P-550-ARI), cuyos contenidos calculados (Bogue) de $\mathrm{C}_{3} \mathrm{~S}-\mathrm{C}_{2} \mathrm{~S}-\mathrm{C}_{3} \mathrm{~A}-\mathrm{C}_{4} \mathrm{AF}$ y $\mathrm{CaSO}_{4}$ son $39,2 \%-25,7 \%-9,9 \%-12,0 \%$ y $6,9 \%$, respectivamente. Las características químicas, físicas, mecánicas y estructurales de dicho cemento se resumen en (2) y su resistencia química (KochSteinegger) frente al agua de mar artificial ASTM D 1141 - 75 y de una disolución saturada de yeso se incluyen en (2) y (4); respectivamente.

b) Disolución de sulfato de sodio, con una concentración de $\mathrm{SO}_{4}(\mathrm{II})=1,42 \mathrm{~g} / \mathrm{I}<>1,48 \times$ $\times 10^{-2}$ moles/litro

Esta disolución se ha preparado disolviendo $2,1 \mathrm{~g}(\times 75)$ de sulfato de sodio comercial en 1 $(\times 75)$ litro de agua desionizada. Posteriormente, se ha determinado la concentración del ion $\mathrm{SO}_{4}$ (II)

La pureza del $\mathrm{Na}_{2} \mathrm{SO}_{4}$ utilizado se ha determinado por vía química y por $\mathrm{DRX}$.

\subsection{Ténica operatoria}

\section{a) Preparación del lecho de cemento hidratado}

A partir del cemento $1<>$ P-550-ARI, objeto del presente estudio, se han preparado dos lechos de cemento hidratado y granulado (uno corresponde a la muestra conservada en la cámara húmeda, a $21 \pm 2^{\circ} \mathrm{C}$ y una humedad relativa superior a $90 \%$, durante 7 días y otro durante 28 días), según se describe en (1).

\section{b) Equipo y procedimiento operatorio}

Se ha empleado un equipo de trabajo análogo al descrito en (1) y utilizado un procedimiento operatorio similar (1).

\section{c) Determinaciones efectuadas}

En cada una de las distintas fracciones recogidas de la disolución de sulfato de sodio, que han atravesado los lechos de cemento, se ha determinado:

c.1. el contenido de los iones $\mathrm{Ca}$ (II) y $\mathrm{SO}_{4}$ (II); el primero por complexometría con EDTA y el segundo por gravimetría, al estado de $\mathrm{BaSO}_{4}$,

c.2. el valor del $\mathrm{pH}$ y de la conductividad, utilizando un $\mathrm{pH}$-metro digital, Crison 501 , con electrodo Metrohm AG, tipo 9100 Herisau y un conductímetro Crison 522, con célula Metrohm CH, tipo 9100 Herisau.

En la pasta granulada de cemento hidratado, antes y después de pasar la disolución, se ha determinado:

c.3. el contenido de los iones $\mathrm{Ca}$ (II) y $\mathrm{SO}_{4}$ (II), de acuerdo con los procedimientos operatorios descritos en el Pliego RC-75 (9).

c.4. la composición estructural por DRX, utilizando un equipo Philips PW-1010 y las condiciones de trabajo que se reseñan en (10).

\section{RESULTADOS}

Sistema: cemento P-550-ARI hidratado-disolución de sulfato de sodio. 


\subsection{Volúmenes extraídos de la disolución de sulfato de sodio}

En la figura 1 se incluyen los volúmenes parciales y totales recogidos de la disolución de sulfato de sodio, que han pasado a través del lecho granulado de cemento, en función del tiempo, correspondientes al cemento hidratadogranulado y conservado durante 7 días. Durante las primeras ocho horas el volumen de las extracciones recogidas cada hora, varía entre 0,12 y 0,21 iitros, el volumen total de la disolución de sulfato de sodio que ha atravesado el lecho ha sido 1,25 litros. En las siguientes 15 horas se ha hecho sólo una extracción, habiéndose recogido 3,03 litros.

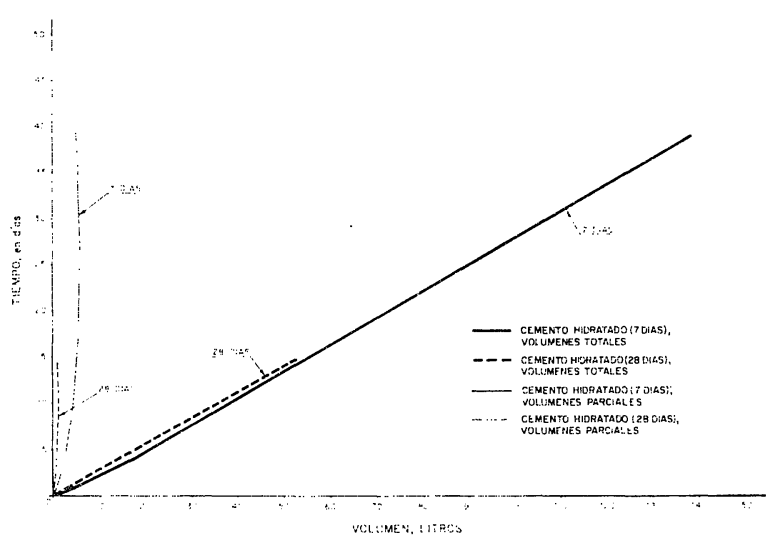

Fig. 1.-Sistemas: cemento 1 hidratado ( 7 y 28 dias)-disolución de sulfato de sodio. Volúmenes parciales y totales en función del tiempo.

A partir de las primeras 24 horas, la cadencia de recogida ha sido de una extracción cada 3-4 horas, 3 veces, y otra en las restantes 15 horas. Después se ha realizado una extracción cada 24-48 horas hasta el final del ensayo 938 horas (39 días).

El volumen total de disolución de sulfato de sodio que ha atravesado el lecho ha sido 137,24 litros.

Así mismo, en dicha figura 1, también se incluyen los volumenes parciales y totales recogidos de la disolución de sulfato de sodio, que ha pasado a través del lecho granulado de cemento hidratado y conservado 28 días.

Durante las ocho primeras extracciones, una cada hora, el volumen recogido varía entre 0,16 y 20 litros, el volumen total de la disolución de sulfato de sodio, que ha atravesado el lecho, ha sido 1,38 litros. En las siguientes 15 horas, el volumen recogido ha sido 2,78 litros.

A lo largo de los siguientes días se han efectuado 4 extracciones ( 1 cada 3 horas, 3 veces, y otra en las 15 horas restantes), habiendo recogido un total de 52,38 litros de la disolución de sulfato de sodio durante 326 horas (13 días); período de tiempo que, a la vista de los resultados obtenidos y que se comentan en los apartados siguientes, se consideró suficiente para este estudio.

\subsection{Variación del contenido de Ca (II)}

Estudio de los valores analíticos.

En la figura 2 se han representado en abscisas las extracciones (volumen acumulado, en litros) y en ordenadas las concentraciones de $\mathrm{Ca}$ (II), en moles/litro $\times 10^{-2}$, presentes en cada una de las extracciones de la disolución de sulfato de sodio, que ha atravesado el lecho granulado de cemento hidratado y curado durante 7 y 28 días.

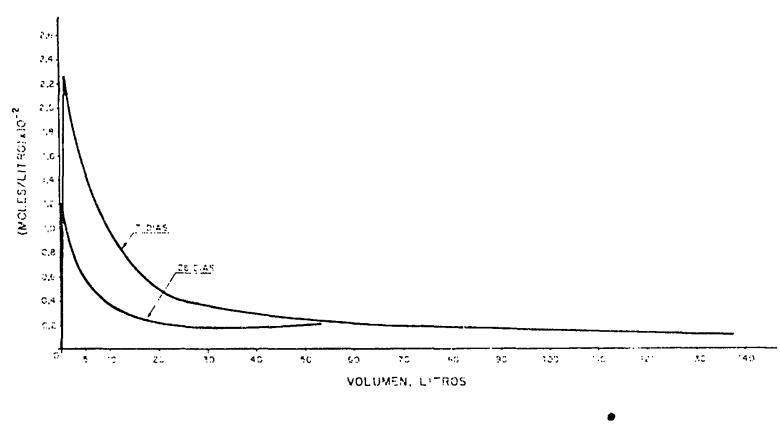

Fig. 2.-Sistemas: cemento 1 hidratado (7 y 28 días)-disolución de sulfato de sodio. Evolución del contenido de ion $\mathrm{Ca}(\mathrm{II})$.

En el lecho del cemento 1, hidratado-granulado y curado durante 7 dias, se observa que en la primera extracción $(0,15$ litros) la concentración de $\mathrm{Ca}$ (II) es $1,0 \times 10^{-2}$ moles/litro, aumentando en las siguientes hasta conseguir el valor máximo $\left(2,2 \times 10^{-2}\right.$ moles/litro), cuando han pasado 1,25 litros de la disolución de sulfato de sodio; a continuación, dicha concentración disminuye exponencialmente hasta alcanzar el valor $0,1 \times 10^{-2}$ moles/litro, cuando se han recogido 116,98 litros.

En el lecho del cemento 1, hidratado-granulado y curado durante 28 días, se aprecia que la concentración de $\mathrm{Ca}$ (II) en la primera extracción (0,17 litros) es $0,9 \times 10^{-2}$ moles/litro; en la siguiente alcanza el valor máximo $1,2 \times 10^{-2}$. moles/litro, cuando han pasado 0,32 litros; después, disminuye exponencialmente hasta conseguir el valor $0,2 \times 10^{-2}$ moles/litro, cuando han atravesado el lecho 25,79 litros de disolución, valor que se mantiene prácticamente constante hasta el final del ensayo. 
En la figura 3 se han representado en abscisas los volúmenes de las extracciones acumulativas (volumen total de la disolución de sulfato de sodio, que ha pasado por cada uno de los lechos fabricados con cemento 1 hidratado, granulado y curado durante 7 y 28 días) y en ordenadas los moles $\times 10^{-1}$ totales de $\mathrm{Ca}$ (II) extraídos. La cantidad de $\mathrm{Ca}$ (II) extraída de ambos lechos de cemento 1 es función del volumen de la disolución de sulfato de sodio que ha atravesado el lecho; esta cantidad aumenta rápidamente en las primeras extracciones $y$, a continuación, se hace ligeramente menor. Dicha cantidad de Ca (II) extraída es mayor en el caso del lecho de cemento hidratadogranulado y curado durante el menor período de tiempo (7 días).

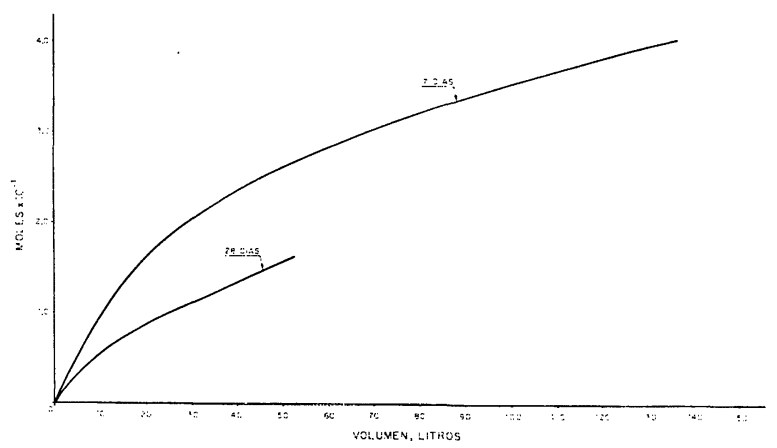

Fig. 3.-Sistemas: cemento 1 hidratado (7 y 28 días)-disolución de sulfato de sodio. Evolución del contenido de ion $\mathrm{Ca}$ (II) extraído, total.

\section{Balance iónico}

En la tabla 1 figuran los contenidos de $\mathrm{Ca}$ (II), en moles, que existen en los $53,48 \mathrm{~g}$ del cemento hidratado y curado durante 7 días puestos inicialmente en el lecho y en los $22,15 \mathrm{~g}$ de cemento del lecho después de pasar a su través 137,2 litros de la disolución de sulfato de sodio; ambas cantidades de cemento están referidas al producto calcinado. Así mismo, en

TABLA 1

Sistema: cemento 1 hidratado

(7 dias)-disolución de sulfato de sodio.

Cantidades de Ca (II), en moles, en los componentes (iniciales y finales)

\begin{tabular}{|l|c|c|c|c|}
\hline \multirow{2}{*}{ Componentes } & \multicolumn{2}{|c|}{ Cantidades } & \multicolumn{2}{c|}{ Ca (II), moles } \\
\cline { 2 - 5 } & iniciales & finales & iniciales & finales \\
\hline Cemento & $53,48 \mathrm{~g}$ & $22,15 \mathrm{~g}$ & 0,57 & 0,11 \\
Dis. de $\mathrm{Na}_{2} \mathrm{SO}_{4}$ & 137,21 & $137,2 \mathrm{I}$ & 0,00 & 0,40 \\
\hline Suma & & & 0,57 & 0,51 \\
\hline
\end{tabular}

dicha tabla, se encuentran los contenidos de dicho ion en el volumen total de la disolución de sulfato de sodio, antes de pasar por el lecho y en el conjunto de las extracciones de dicha disolución una vez que han atravesado el lecho de cemento hidratado, granulado y curado durante 7 días.

En la tabla 2 se incluyen los contenidos del ion $\mathrm{Ca}$ (II) en los $53,48 \mathrm{~g}$ y $41,62 \mathrm{~g}$ de cemento del lecho fabricado con cemento hidratado, granulado y curado durante 28 días, antes y después de ser sometidos a la acción de 52,38 litros de la disolución de sulfato de sodio; dichas cantidades de cemento están referidas al producto calcinado.

TABLA 2

Sistema: cemento 1 hidratado

(28 dias)-disolución de sulfato de sodio.

Cantidades de Ca (II), en moles, en los componentes (iniciales y finales)

\begin{tabular}{|l|c|c|c|c|}
\hline \multirow{2}{*}{ Componentes } & \multicolumn{2}{|c|}{ Cantidades } & \multicolumn{2}{c|}{ Ca (II), moles } \\
\cline { 2 - 5 } & iniciales & finales & iniciales & finales \\
\hline Cemento & $53,48 \mathrm{~g}$ & $41,62 \mathrm{~g}$ & 0,57 & 0,40 \\
Dis. de $\mathrm{Na}_{2} \mathrm{SO}_{4}$ & $52,38 \mathrm{I}$ & $52,38 \mathrm{I}$ & 0,00 & 0,16 \\
\hline Suma & & 0,57 & 0,56 \\
\hline
\end{tabular}

En ambas tablas se puede observar que en el primer sistema (tabla 1), aproximadamente, el $80,7 \%$ del ion $\mathrm{Ca}$ (II) presente en el cemento inicial del lecho $(0,57$ moles) ha pasado a los 137,2 litros de la disolución de sulfato de sodio, en donde se han encontrado 0,40 moles; el resto de dicho ion $\mathrm{Ca}$ (II) $(0,11$ moles) ha quedado en el cemento. La extracción por litro de disolución ha sido 0,003 moles. En el segundo sistema (tabla 2), que corresponde al cemento hidratado, granulado y curado durante 28 días, solo se ha extraído el 29,8 \% del contenido total de ion $\mathrm{Ca}$ (II) por los 52,38 litros de la disolución de sulfato de sodio; la cantidad de $\mathrm{Ca}$ (II) extraída $(0,16$ moles por los 52,38 litros de dicha disolución) por litro de disolución es análoga a la del sistema anterior $(0,003$ moles/litro).

\subsection{Variación del contenido de $\mathrm{SO}_{4}$ (II)}

Estudio de los valores analíticos

En la figura 4 se han representado en abscisas las extracciones (volumen acumulado, en litros) y en ordenadas las concentraciones de 
$\mathrm{SO}_{4}$ (II) en moles/litro $\times 10^{-3}$, presentes en cada una de las extracciones efectuadas en los lechos de cemento hidratado-granulado y curado durante 7 y 28 días.

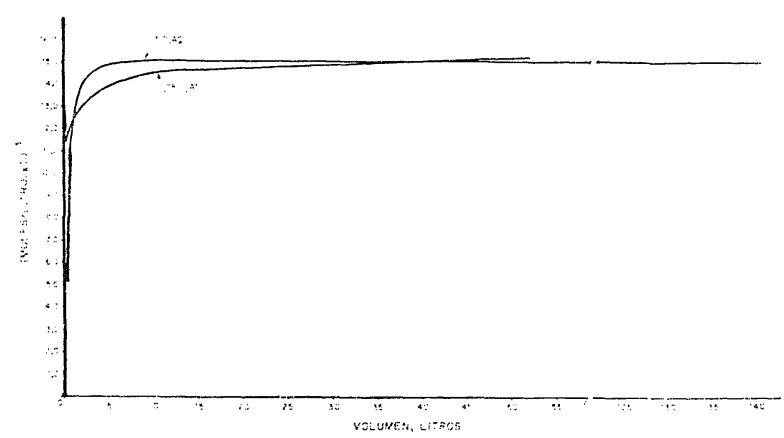

Fig. 4.-Sistemas: cemento 1 hidratado (7 y 28 días)-disolución de sulfato de sodio. Evolución del contenido de ion $\mathrm{SO}_{4}(\mathrm{II})$.

En el caso del sistema 1 hidratado (7 días)disolución de sulfato de sodio se observa que en la primera extracción ( 0,15 litros) la concentración de $\mathrm{SO}_{4}$ (II) es 5,2 $\times 10^{-3}$ moles/litro, aumentando rápidamente hasta alcanzar el valor $15 \times 10^{-3}$ moles/litro (valor análogo al de la disolución de sulfato de sodio) cuando han pasado 4,71 litros de dicha disolución de sulfato de sodio a través del lecho de cemento; a partir de este momento permanece prácticamente constante hasta- el final del ensayo.

La concentración de $\mathrm{SO}_{4}$ (II) en la primera extracción (0,17 litros) del lecho de cemento hidratado-granulado y curado durante 28 días, es $11,7 \times 10^{-3}$ moles/litro; a continuación, como en el caso anterior, aumenta rápidamente dicha concentración de $\mathrm{SO}_{4}$ (II) hasta alcanzar el valor $15,0 \times 10^{-3}$ moles/litro en la extracción n. ${ }^{\circ} 20$, cuando han pasado 6,53 litros de la disolución de sulfato de sodio a través del lecho, valor que se mantiene prácticamente constante hasta el final del ensayo.

En la figura 5 se han representado en abscisas las extracciones acumulativas (volumen total, en litros, de la disolución de sulfato de sodio que han pasado por los lechos) y en ordenadas los moles $\times 10^{-1}$ totales, en las extracciones, de $\mathrm{SO}_{4}$ (II), en donde se puede apreciar que la cantidad total de $\mathrm{SO}_{4}$ (II) de las extracciones crece linealmente conforme lo hace el volumen de la disolución de sulfato de sodio, que atraviesa los dos lechos de cemento hidratado-granulado y curado durante 7 ó 28 días. La cantidad total de $\mathrm{SO}_{4}$ (II) es ligeramente inferior en el sistema correspondiente al cemento hidratado durante menor período de tiempo.

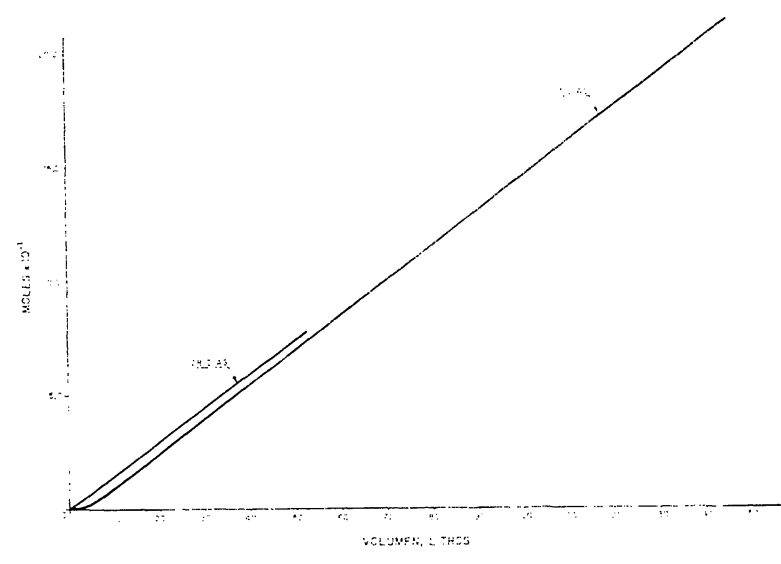

Fig. 5.-Sistemas: cemento 1 hidratado (7 y 28 dias)-disolución de sulfato de sodio. Evolución del contenido total de ion $\mathrm{SO}_{4}$ (II) en las extracciones.

\section{Balance iónico}

En la tabla 3 figuran los contenidos de $\mathrm{SO}_{4}$ (II), en moles, que existen en los $53,48 \mathrm{~g}$ y $22,15 \mathrm{~g}$ de cemento, referidos al producto calcinado, puestos inicialmente en el lecho y los que existen una vez que han pasado 137,2 litros de la disolución de sulfato de sodio; así mismo, se encuentran los contenidos de dicho ion $\mathrm{SO}_{4}$ (II) en los 137,2 litros de la mencionada disolución, antes de pasar por el lecho, y en el conjunto de las diversas fracciones recogidas cuando han atravesado el lecho del sistema cemento 1 hidratado (7 días)-disolución de sulfato de sodio.

\section{TABLA 3}

Sistema: cemento 1 hidratado

(7 días)-disolución de sulfato de sodio. Cantidades de $\mathrm{SO}_{4}(I I)$, en moles, en los componentes (iniciales y finales)

\begin{tabular}{|l|c|c|c|c|}
\hline \multirow{2}{*}{ Componentes } & \multicolumn{2}{|c|}{ Cantidades } & \multicolumn{2}{c|}{ SO $_{4}$ (II), moles } \\
\cline { 2 - 5 } & iniciales & finales & iniciales & finales \\
\hline Cemento & $53,48 \mathrm{~g}$ & $22,15 \mathrm{~g}$ & $2,65 \times 10^{-2}$ & $0,70 \times 10^{-2}$ \\
Dis. de $\mathrm{Na}_{2} \mathrm{SO}_{4}$ & $137,2 \mathrm{I}$ & 137,21 & 2,03 & 2,01 \\
\hline Suma & & 2,06 & 2,02 \\
\hline
\end{tabular}

En la tabla 4 se incluyen los contenidos de ion $\mathrm{SO}_{4}$ (II) tanto en los $53,48 \mathrm{~g}$ y $41,62 \mathrm{~g}$ de cemento del lecho, referidos al producto calcinado antes y después de pasar por dicho lecho 52,38 litros de la disolución de sulfato de sodio, como en dicha disolución antes de pasar por el lecho y en el conjunto de las fracciones recogidas (52,38 litros, en total) una 
vez que han atravesado el lecho del sistema cemento 2 hidratado (28 días)-disolución de sulfato de sodio.

\section{TABLA 4}

Sistema: cemento 1 hidratado

(28 dias)-disolución de sulfato de sodio.

Cantidades de $\mathrm{SO}_{4}($ II), en moles, en los componentes (iniciales y finales)

\begin{tabular}{|c|c|c|c|c|}
\hline \multirow[b]{2}{*}{ Componentes } & \multicolumn{2}{|c|}{ Cantidades } & \multicolumn{2}{|c|}{$\mathrm{SO}_{4}$ (II), moles } \\
\hline & iniciales & finales & iniciales & finales \\
\hline Cemento & $53,48 \mathrm{~g}$ & $41,62 \mathrm{~g}$ & $2.65 \times 10^{-2}$ & $4.17 \times 10^{-2}$ \\
\hline Dis. de $\mathrm{Na}_{2} \mathrm{SO}_{4}$ & 52.381 & 52,381 & $77,5 \times 10^{-2}$ & $76,5 \times 10^{-2}$ \\
\hline Suma & & & $80,2 \times 10^{-2}$ & $80,7 \times 10^{-2}$ \\
\hline
\end{tabular}

En ambas tablas, se puede observar que existe una disminución del contenido del ion $\mathrm{SO}_{4}$ (II) en el cemento del lecho de $1,95 \times 10^{-2}$ moles en el primer sistema, por donde han pasado 137,2 litros de la disolución de sulfato de sodio y un incremento de $1,52 \times 10^{-2}$ moles en el segundo sistema, por donde han pasado 52,38 litros de dicha disolución.

\subsection{Variación del $\mathrm{pH}$ y de la conductividad}

En la figura 6 se han representado los valores del $\mathrm{pH}$ y de la conductividad $(\mathrm{mho} / \mathrm{cm}) \times 10^{-3}$ en ordenadas, de la disolución de sulfato de sodio, en función del volumen de dicha disolucio (litros), que ha atravesado e! lecho de cemento hidratado y gran!ıladı, en abscisas.

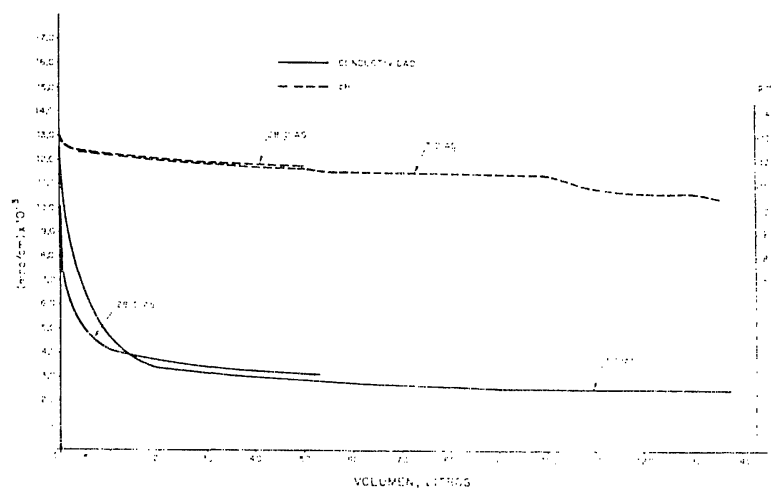

Fig. 6.-Sistemas: cemento 1 hidratado ( 7 y 28 días)-disolución de sulfato de sodio. Evolución del phi y de la conductividad.

\section{Variación del pH}

En ambos sistemas [cemento 1 hidratado (7 dias)-disolución de sulfato de sodio y cemento 1 hidratado (28 días)-disolución de sulfato de sodio], el pH sigue una evolución análoga, su valor está comprendido entre 12,7 y 10,3 para el primer sistema y entre 12,9 y 11,6 para el segundo.

\section{Variación de la conductividad}

La conductividad de la disolución de sulfato de sodio $\left(2,9 \times 10^{-3} \mathrm{mho} / \mathrm{cm}\right)$ experimenta un aumento considerable cuando han pasado las primeras cantidades $(0,15$ y 0,17 litros $)$ a través de los lechos de cemento, alcanzando los valores $16,9 \times 10^{-3} \mathrm{mho} / \mathrm{cm}$ y $12,7 \times 10^{-3}$ mho/cm para ambos sistemas (cemento hidratado y curado durante 7 y 28 dias respectivamente). A continuación, se produce una disminución exponencial, obteniéndose, para los dos sistemas, el valor $3,5 \times 10^{-3} \mathrm{mho} / \mathrm{cm}$ cuando han pasado 17,0 y 25,0 litros, respectivamente; este valor se conserva prácticamente constante para el primer sistema y disminuye ligeramente para el segundo, llegando a ser $2,5 \times 10^{-3} \mathrm{mho} / \mathrm{cm}$.

\subsection{Estudio por difracción de rayos $X$ del cemento hidratado e hidratado ataca- do de los lechos}

En la figura 7 se incluyen los DRX del cemen-
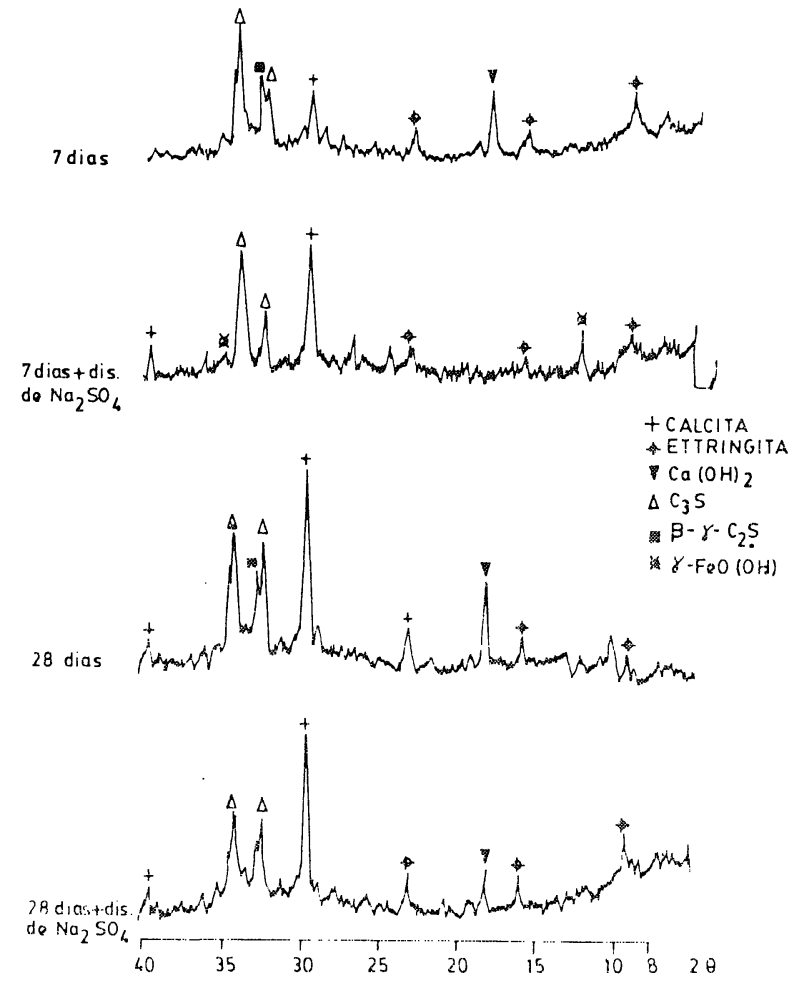

Fig. 7.--Sistemas: cemento 1 hidratado (7 y 28 dias)-disolución de suifato de sodio. DRX de los cementos hidratacos e hidratados-atacados. 
to 1 hidratado-conservado durante 7 días y cemento 1 hidratado-conservado durante 28 días, antes y después de someterlos a la acción de la disolución de sulfato de sodio. En dichos difractogramas se han detectado los picos de los siguientes compuestos cristalinos:

a) Diagramas de difracción de rayos $X$ del cemento 1 hidratado (7 y 28 dias).

- Ettringita: los picos de este compuesto tienen una intensidad análoga en ambos DRX.

- Portlandita: la intensidad de los picos en el DRX del cemento hidratado y curado durante 28 días es 1,25 veces mayor que la del DRX del cemento hidratado y curado 7 dias, como consecuencia del avance de las reacciones de hidratación.

- Calcita: la intensidad de los picos en el DRX del cemento hidratado y curado durante 28 días es 3 veces mayor que la del DRX del cemento hidratado y curado 7 días.

- Compuestos anhidros del cemento (fundamentalmente $\mathrm{C}_{3} \mathrm{~S}$ y $\mathrm{C}_{2} \mathrm{~S}$ ), cuyos picos presentan intensidad análoga en los dos diagramas.

b) Diagramas de difracción de rayos $X$ del cemento 1 hidratado (7 y 28 días) sometido a la acción de la disolución de sulfato de sodio.

- Ettringita: los picos de este compuesto se han detectado en los DRX de las muestras de los dos lechos. Su intensidad es lig ramente inferior en el diagrama del cemento hidratado y curado durante 7 días, sometido a la acción de la disolución de sulfato de sodio, que en el de la muestra hidratada sin someter a la acción de dicha disolución; por el contrario, dicha intensidad en el DRX de la muestra de cemento hidratado y curado durante 28 días, sometido a la acción de la disolución mencionada, es ligeramente superior que el correspondiente a la muestra de cemento hidratado y curado durante 28 días antes de someterla a la acción de esta disolución.

- Portiandita: los picos de la portlandita, de intensidad menor que en el diagrama de la muestra correspondiente antes de someterla a la acción de la disolución de sulfato de sodio, se han detectado en el DRX de la muestra del lecho de cemento hidratado (28 dias) por el que han pasado 52,38 litros de la disolución de sulfato de sodio. Por el contrario, en el DRX de la muestra del lecho de cemento hidratado (7 días), someti- do a la acción de la disolución de sulfato de sodio (137,2 litros), han desaparecido.

- Calcita: la intensidad de los picos de este compuesto en el diagrama del cemento hidratado y curado durante 28 dias, sometido a la acción de la disolución de sulfato de sodio, es mayor que el análogo de la muestra de cemento hidratada y curada durante 7 dias.

La intensidad de los picos de la calcita de la muestra de cemento hidratado ( 7 días), sometido a la acción de la disolución de sulfato de sodio, es mayor que en el DRX del cemento antes de someterlo a dicha acción, mientras que para la muestra hidratada (28 dias), sometida a la acción de la disolución de sulfato de sodio es ligeramente menor.

- Compuestos anhidros del cemento (fundamentalmente $\mathrm{C}_{3} \mathrm{~S}$ y $\mathrm{C}_{2} \mathrm{~S}$ ): sus picos son de menor intensidad que en los DRX del cemento 1 hidratado ( 7 y 28 dias).

- Los picos del compuesto $\gamma-\mathrm{FeO}(\mathrm{OH})$ aparecen sólo en la muestra correspondiente al cemento hidratado o curado durante 7 días por el que han pasado 137,2 litros de la disolución de sulfato de sodio.

\section{INTERPRETACION DE RESULTADOS}

El Ca (II) que aparece en las diversas extracciones de la disolución de sulfato de sodio, que han atravesado los lechos de cemento 1 hidratado y granulado, procede, de un modo especial, del $\mathrm{Ca}(\mathrm{OH})_{2}$ formado durante las reacciones de hidratación del $\mathrm{C}_{3} \mathrm{~S}$ y $\mathrm{C}_{2} \mathrm{~S}$ de la fracción clínker del cemento, así como del $\mathrm{CaO}$ libre, en virtud de la reacción $(A)$ :

(A)

$$
\begin{gathered}
\mathrm{Ca}(\mathrm{OH})_{2} \text {.sólido }+ \text { dis. } \mathrm{Na}_{2} \mathrm{SO}_{4} \rightleftharpoons \\
=\mathrm{Ca}(\mathrm{OH})_{2} \text {.disuelto }=\frac{\mathrm{Ca}(\mathrm{II})+2 \mathrm{OH}(\mathrm{I})}{\text { disolución }}
\end{gathered}
$$

producciendo un incremento brusco del $\mathrm{pH}$ del medio.

La solubilidad del hidróxido de calcio ( $\mathrm{pK}=$ $=5,3$ ) en agua es $1,230 \mathrm{~g} / \mathrm{l}$, a $20^{\circ} \mathrm{C}$, según Biczok (11), ó 1,23 g/l como $\mathrm{CaO}<>1,625 \mathrm{~g} / \mathrm{l}$ como $\mathrm{Ca}(\mathrm{OH})_{2}$, según Duriez y Arrambadi (12), mientras que la de los silicatos de calcio hidratados es $50 \mathrm{mg} / \mathrm{l}$ como $\mathrm{CaO}(13)(14)<>66$ $\mathrm{mg} / \mathrm{l}$ como $\mathrm{Ca}(\mathrm{OH})_{2}$. La ettringita se disuelve ligeramente con separación de gel de alúmina, sulfato de calcio dihidratado e hidróxido de calcio, según ciertos autores (15) (16), y es extraordinariamente poco soluble $(0,001 \mathrm{~g}$ de 
$\mathrm{SO}_{3}$ por $100 \mathrm{ml}$ de agua saturada de cal), según otros (17).

En el sistema cemento 1 hidratado (7 días)disolución de sulfato de sodio se ha obtenido la máxima cantidad de $\mathrm{Ca}$ (II) extraído del lecho $\left(2,25 \times 10^{-2}\right.$ moles/litro), que se corresponde con la solubilidad teórica en agua, cuando han pasado 0,91 litros de la disolución de sulfato de sodio a través de dicho lecho; sin embargo, en el sistema cemento 1 hidratado (28 días)-disolución de sulfato de sodio, la máxima cantidad de $\mathrm{Ca}$ (II) extraída $\left(1,24 \times 10^{-2}\right.$ moles/litro, inferior a la del sistema anterior) se ha conseguido cuando han pasado por el lecho 0,32 litros.

$\mathrm{El} \mathrm{CO}_{2}$ disuelto en la disolución de sulfato de sodio es capaz de reaccionar, en medio básico fuerte, con el $\mathrm{Ca}(\mathrm{OH})_{2}$ presente en el cemento hidratado del lecho formando calcita, como se ha puesto de manifiesto en los DRX correspondientes, produciendo además una disminución del $\mathrm{pH}$. La reacción que tiene lugar es:

$$
\begin{gathered}
\mathrm{Ca}(\mathrm{OH})_{2} \text {. sólido } \rightleftharpoons \mathrm{Ca}(\mathrm{OH})_{2} \text {.disuelto } \rightleftharpoons \\
\begin{array}{c}
\rightleftharpoons \mathrm{Ca}(\mathrm{II})+2 \mathrm{OH}(\mathrm{I}) \\
+ \\
\mathrm{CO}_{2} \\
1 \\
\mathrm{CaCO}_{3} \text {.sólido }+\mathrm{H}_{2} \mathrm{O}
\end{array}
\end{gathered}
$$

La concentración del ión $\mathrm{SO}_{4}$ (II) de la disolución de sulfato de sodio $\left(1,48 \times 10^{-2}\right.$ moles/litro) alcanza su valor mínimo cuando la segunda cantidad $(0,27$ litros y 0,32 litros para el cemento hidratado y curado 7 y 28 dias, respectivamente), correspondiente a la segunda extracción, ha atravesado el lecho de ambos sistemas; dichas concentraciones son $0,47 \times$ $\times 10^{-2}$ moles/litro (primer sistema) y $1,12 \times$ $\times 10^{-2}$ moles/litro (segundo sistema). En las extracciones siguientes, la concentración del mencionado ion $\mathrm{SO}_{4}$ (II) de la disolución inicial experimenta progresivamente una disminución menor hasta que, dicha concentración, se mantiene, prácticamente, constante; los valores son próximos a $1,48 \times 10^{-2}$ moles/litro cuando han pasado por el lecho 4,71 y 28,29 litros de la disolución para el primero y el segundo sistema, respectivamente. En resumen, en un principio parte de la concentración del ion $\mathrm{SO}_{4}$ (II), en pequeñas cantidades, es retenido por el cemento 1 hidratado de los lechos (dadas las características estructurales del cemento), formando ettringita según la reacción (D), como se ha puesto de manifiesto por DRX.

Para que esta formación de ettringita tenga lugar, es necesario que los iones $\mathrm{SO}_{4}$ (II) reaccionen con los iones $\mathrm{Ca}$ (II) del hidróxido, en una primera etapa, en la interfase $\mathrm{Ca}(\mathrm{OH})_{2}$.sóli- do $=\mathrm{Ca}(\mathrm{OH})_{2}$.disuelto, según $(\mathrm{C})$ :

(C)

$$
\begin{aligned}
& \mathrm{Ca}(\mathrm{OH})_{2} \text {. sólido }\left[\rightleftharpoons \mathrm{Ca}(\mathrm{OH})_{2} \text {.disuelto }\right]+ \\
& +\mathrm{SO}_{4}(\mathrm{II}) \stackrel{\mathrm{H}_{2} \mathrm{O}}{=} \mathrm{CaSO}_{4} \cdot 2 \mathrm{H}_{2} \mathrm{O} \text {.sólido }
\end{aligned}
$$

obteniéndose $\mathrm{CaSO}_{4} .2 \mathrm{H}_{2} \mathrm{O}$ que, posteriormente, forma ettringita, según (D):

$$
\text { (D) } \begin{array}{ll} 
& 3 \mathrm{CaO} \cdot \mathrm{Al}_{2} \mathrm{O}_{3} \cdot 6 \mathrm{H}_{2} \mathrm{O}+3\left(\mathrm{CaSO}_{4} \cdot 2 \mathrm{H}_{2} \mathrm{O}\right)+ \\
+ & 19 \mathrm{H}_{2} \mathrm{O} \rightleftharpoons \\
= & 3 \mathrm{CaO} \cdot \mathrm{Al}_{2} \mathrm{O}_{3} \cdot 3 \mathrm{CaSO}_{4} \cdot 31 \mathrm{H}_{2} \mathrm{O}
\end{array}
$$

de aquí que, la formación del yeso tenga lugar en los primeros momentos, cuando la cantidad de $\mathrm{Ca}(\mathrm{OH})_{2}$ en el lecho es mayor y antes de que se disuelva y arrastre en la disolución de sulfato de sodio.

El $\mathrm{pH}$ de las distintas fracciones recogidas de la disolución de sulfato de sodio, que han atravesado los lechos de cemento, viene regido por el equilibrio (A). En la primera extracción, para ambos sistemas, se observa un incremento de 5 unidades con relación al $\mathrm{pH}$ de la disolución; el valor del $\mathrm{pH}$ es 12,7 para el primer sistema ( 7 dias) y 12,9 para el segundo (28 días), valores que a lo largo del ensayo descienden hasta 10,3 y 11,6, respectivamente.

En cuanto a la conductividad, que es función de la cantidad de los iones presentes en la disolución, pasa de $2,9 \times 10^{-3} \mathrm{mho} / \mathrm{cm}$, valor de la disolución de sulfato de sodio, a 16,96 $\times$ $\times 10^{-3} \mathrm{mho} / \mathrm{cm}$ y $12,70 \times 10^{-3} \mathrm{mho} / \mathrm{cm}$ para el primero y el segundo sistema, respectivamente, en la primera extracción; este incremento se debe, de un modo especial, a la presencia en la disolución de los iones $\mathrm{Ca}$ (II) y $\mathrm{OH}$ (I) procedentes del equilibrio (A). A continuación, la conductividad disminuye exponencialmente del mismo modo que lo hacen las concentraciones de $\mathrm{Ca}$ (II) y de $\mathrm{OH}(\mathrm{I})$.

Teniendo en cuenta que las conductividades iónicas equivalentes, a dilución infinita y a $25^{\circ} \mathrm{C}$, expresadas en $\mathrm{ohm}^{-1} \cdot \mathrm{cm}^{2}$. equiv. ${ }^{-1}$, de los iones $\mathrm{Na}$ (I), $\mathrm{Ca}$ (II), $\mathrm{OH}$ (I) y $\mathrm{SO}_{4}$ (II) son: 50,1 - 59,5 - 197,6 y 80,0 (18), se considera que en la evolución de las conductividades de las fracciones recogidas de la disolución de sulfato de sodio influye el equilibrio (A) y, especialmente, la concentración de los iones $\mathrm{OH}$ (I). Como puede apreciarse la evolución de las conductividades permite seguir, más claramente que la del $\mathrm{pH}$, los diferentes estados del equilibrio (A).

La disolución progresiva del $\mathrm{Ca}(\mathrm{OH})_{2}$, electrolito débil, en las fracciones de la disolución de sulfato de sodio que atraviesa los lechos de cemento, favorece las reacciones de hidratación de la fracción clínker del cemento, liberando más portlandita que pasa a la disolución 
en forma iónica llegando, prácticamente, a. desaparecer de los lechos de cemento, a la vez que los silicatos se degradan; parte de esta portlandita ha reaccionado con el $\mathrm{CO}_{2}$ y con el $\mathrm{SO}_{4}$ (II) de la disolución para dar pequeñas cantidades de calcita y de yeso. Fenómenos que se han puesto de manifiesto por DRX.

Como consecuencia de los fenómenos de disolución mencionados, que tienen lugar en estos sistemas: cemento 1 ( 7 y 28 días)-disolución de sulfato de sodio, análogos a los de los sistemas: cemento 1 ( 7 y 28 días)-agua desionizada (1), se produce una pérdida de masa del lecho de cemento hidratado que es función de! tiempo de curado y. muy especialmente, del volumen de la disolución que ha atravesado el lecho. Así, las cantidades de cemento hidratado de los lechos estudiados han experimentado una disminución del $58,6 \%$ en el sistema cemento 1 hidratado ( 7 dias)-disolución de sulfato de sodio y del $31,2 \%$ en el sistema cemento 1 hidratado (28 días)-disolución de sultato de sodio por los que han pasado 137,2 litros y 52,38 litros de dicha disolución, respectivamente. Estas cantidades, por litro de disolución y para cada lecho, son del mismo orden.

\section{CONCLUSIONES}

\section{Primera}

El hidróxido de calcio que se encuentra en los lechos de cemento 1 hidratado y granulado, que procede de las reacciones de hidratación de la fracción clínker, se disuelve - total o parcialmente- en la disolución acuosa de sulfato de sodio que atraviesa dichos lechos. Las cantidades disueltas de $\mathrm{Ca}(\mathrm{OH})_{2}$ experimentan una disminución exponencial conforme aumenta el volumen de la disolución de sulfato de sodio, que ha pasado por los lechos de cemento.

\section{Segunda}

La concentración de los iones sulfato de la disolución en los primeros volúmenes que pasan por los lechos de cemento (las dos primeras extracciones, 0,59 litros en total), experimenta una disminución al ser retenidos por los compuestos del cemento, dando las sales de calcio correspondientes. Esta disminución de iones $\mathrm{SO}_{4}$ (II) se hace paulatinamente menor conforme aumenta el volumen de la disolución que pasa por los lechos, hasta que llega un momento en el cual la concentración de dichos iones no experimenta, prácticamente, variación.

\section{Tercera}

Tanto la cantidad de $\mathrm{Ca}$ (II) en las diversas fracciones recogidas de la disolución de sulfato de sodio, que han atravesado los lechos de cemento, como la de $\mathrm{SO}_{4}$ (II) retenidos por el cemento hidratado, son función del tiempo de curado de dichos cementos y, fundamentalmente, del volumen de la disolución acuosa de sulfato de sodio puesto en juego.

El cemento hidratado de los lechos, sometidos a la acción de la disolución de sulfato de sodio, experimenta una disminución considerable de masa, que depende, de un modo especial, del volumen de dicha disolución que ha pasado por los lechos; así, en el cemento 1 hidratado y curado durante 7 días, por el que han pasado 137,2 I de la disolución de sulfato de sodio, se produce una pérdida de masa del $58,4 \%$, mientras que en el cemento 1 hidratado y curado durante 28 días, por el que han pasado 52,38 I de la disolución mencionada, es del $24,3 \%$.

\section{Cuarta}

La conductividad de las distintas fracciones recogidas de la disolución de sulfato de sodio, que han atravesado los lechos de cemento, experimenta un incremento brusco en la primera extracción $(0,15$ I) y, a continuación, una disminución exponencial conforme aumenta el volumen de dicha disolución. La evolución de la conductividad, análoga a la de la disolución de $\mathrm{Ca}(\mathrm{OH})_{2}$, es función, principalmente, de la concentración de iones $\mathrm{OH}$ (I) presentes en dicha disolución que, a su vez, depende de la cantidad disuelta de hidróxido de calcio.

Así mismo, el valor del $\mathrm{pH}$ de las mencionadas fracciones recogidas de la disolución de sulfato de sodio es función de la cantidad de $\mathrm{Ca}(\mathrm{OH})_{2}$ disuelto; los valores máximos $(\mathrm{pH}=$ $=12,2$ a 12,5) se obtienen en la primera extracción y los menores $(\mathrm{pH}=11,6$ a 10,3$)$ en las últimas.

\section{Quinta}

Los compuestos cristalinos del cemento hidratado (de un modo especial ettringita, portlandita y calcita) y de la fracción clinker anhidra, que se han sometido a la acción de la disolución de sulfato de sodio, experimentan una modificación considerable. Así, en los DRX obtenidos, la intensidad de los picos de la portlandita ha disminuido, llegando a desaparecer cuando han pasado por el lecho 137,2 I de la disolución y los de la calcita se han incrementado; los picos de la ettringita son ligeramente inferiores o superiores según el tiempo de curado del cemento hidratado y del volumen de la disclución que ha atravesado el lecho. 


\section{BIBLIOGRAFIA}

(1) BERMEJO-MUÑOZ, M. ${ }^{a}$ F., SAGRERA-MORENO, J. L. y GASPAR-TEBAR, D. (1987): Resistencia química del hormigón. XXVII. Contribución al estudio del sistema: cemento P-550-ARI hidratado-agua desionizada. Materiales de Construcción, 205, 37-47.

(2) SAGRERA-MORENO, J. L. y GASPAR-TEBAR, D. (1980): Resistencia quimica del hormigón. IV. Acción del agua de mar: influencia de la adición de escoria a un cemento portland de alta resistencia inicial. Evolución de las resistencias mecánicas a flexotracción y del coeficiente de corrosión. Materiales de Construcción, 178, 17-38.

(3) GASPAR-TEBAR, D. y SAGRERA-MORENO, J. L. (1981): Resistencia química del hormigón. IX. Influencia de la adición de escoria a un cemento portland de alta resistencia inicial. Estudio por DRX del sistema: cemento 1/escoria-agua potable filtrada. Materiales de Construcción, 181, 33-44.

(4) SAGRERA-MORENO, J. L. y GASPAR-TEBAR, D. (1982): Resistencia química del hormigón. XV. Acción de una disolución saturada de yeso: influencia de la adición de escoria a un cemento portland de alta resistencia inicial. Evolución de las resistencias mecánicas a flexotracción y de los coeficientes de corrosión. Materiales de Construcción, 185. 29-42.

(5) GASPAR-TEBAR, D. y SAGRERA-MORENO, J. L. (1982): Resistencia química del hormigón. XVI. Acción del agua de mar: influencia de la adición de escoria a un cemento portland de alta resistencia inicial. Estudio por DRX. Materiales de Construcción, 186, 39-60.

(6) SAGRERA-MORENO, J L y GASPAR-TEBAR, D. (1983): Resistencia química del hormigón. XXI. Influencia de la adición de escoria a un cemento portland de alta resistencia inicial. Estudio de la concentración iónica del sistema: cemento 1/escoria-agua potable filtrada. Materiales de Construcción, 192, 33-41.

(7) GASPAR-TEBAR, D. y SAGRERA-MORENO, J. L. (1984): Resistencia química del hormigón. XXII. Influencia de la adición de escoria a un cemento portland de alta resistencia inicial. Estudio de la concentración iónica del sistema: cemento 1/escoria-agua de mar artificial. Materiales de Construcción, 193, 51-68.

(8) DIAZ-MORA, R., SAGRERA-MORENO, J. L. y GASPAR-TEBAR, D. (1980): Resistencia química del hormigón. VI. Acción del agua de mar artificial que fluye a través de un lecho de mortero fabricado con un cemento portland de alta resistencia inicial. Materiales de Construcción, 179, 81-99.

(9) Pliego de Prescripciones Técnicas Generales para la Recepción de Cementos, 1975 (RC-75): MOP, Servicio de Publicaciones, Madrid, 1975.

(10) GASPAR-TEBAR, D., SAGRERA-MORENO, J. L. y BERMEJO-MUÑOZ, M. ${ }^{a}$ F. (1981): Resistencia química del hormigón. XI. Contribución al estudio del sistema: cemento P-450-Y-agua desionizada. Materiales de Construcción, 183, 17-30.

(11) BICZOK, I. (1972): Corrosión y Protección del Hormigón. Edit. Urmo, Bilbao, pág. 211.

(12) DURIEZ, M. et ARRAMBADI, J. (1961): Nouv. Trait. Mat. Const., Tome 1. Edit. Dunod, París, págs. 439-440.

(13) ALEGRE, R. (1978): Comportement des ciments en milieu agresiff. Annales de l'Inst. Techn. de Bât. et des Trav. Publ.; serie: Sols et Fondations, n. ${ }^{159}$, 364, 78.

(14) DREYFUS, J. (1960): La Chimie des Ciments. Edit. Eyrolles; París, Tome I, pág. 41.

(15) TAYLOR, H. F. W. (1964): The Chemistry of Cements. Acad. Press. Inc.; London and New York, vol. 1, pág. 261.

(16) LEA, F. M. y DESCH. E. H. (1960): Química del Cemento y del Hormigón. Tipografía Artística; Madrid, pág. 228.

(17) BOGUE, R. H. (1962): La Química del Cemento Portland. Edit. Dossat; Madrid, pág. 600.

(18) CHARLOT, G. (1963): Química Analítica General. Tomo II. Métodos electroquímicos y absorciométricos, cromatografía. Edit. Toray Masson, Barcelona, págs. 123-124.

\section{RECONOCIMIENTO}

Nuestro más sincero agradecimiento a las personas del Equipo de Durabilidad del IETcc: Amalia Rodríguez Pereira, Lucila López Solana, M. ${ }^{a}$ Soledad Cid Fernández, Felipe Cantero Palacios y Manuel Cantero Palacios, así como a M. ${ }^{a}$ Isabel Jiménez, por su valiosa colaboración en la realización de este trabajo. 